\title{
A Complex for Diagnostics of Laser Radiation of Semiconductor Targets Excited by a High-Frequency-Modulated Electron Beam
}

\author{
A. S. Nasibov ${ }^{a} *$, K. V. Berezhnoi ${ }^{a}$, I. D. Tasmagulov ${ }^{a}$, M. I. Yalandin ${ }^{b}$, \\ A. G. Sadykova ${ }^{b}$, M. R. Ul'maskulov ${ }^{b}$, and S. A. Shunailov ${ }^{b}$ \\ ${ }^{a}$ Lebedev Physics Institute, Russian Academy of Sciences, Moscow, 119999 Russia \\ ${ }^{b}$ Institute of Electrophysics, Ural Branch, Russian Academy of Sciences, \\ Yekaterinburg, 620016 Russia \\ *e-mail: nas2121@mail.ru \\ Received April 3, 2019; revised April 15, 2019; accepted April 21, 2019
}

\begin{abstract}
A complex of equipment for diagnosing the parameters of laser radiation of semiconductor targets, which are excited by high-frequency-modulated electron-beam pulses, is described. The ability to control the shape and duration of laser radiation in the picosecond range via high-frequency modulation of an accelerating-voltage pulse was demonstrated experimentally. A train of laser-radiation pulses with a maximum intensity of up to $2 \times 10^{7} \mathrm{~W} / \mathrm{cm}^{2}$ was obtained on a cadmium sulfide target $(\lambda=525 \mathrm{~nm})$.
\end{abstract}

DOI: $10.1134 / \mathrm{S} 002044121905021 \mathrm{X}$

The possibility of obtaining laser radiation from semiconductors that are excited by a strong pulsed electric field or accelerated electrons was first substantiated by N.G. Basov, B.M. Vul, and Yu.M. Popov and then used in studies by Russian and foreign scientists (see, e.g., monographs [1, 2]). Despite the achieved successes in theoretical and experimental studies, the features of lasing of semiconductor targets (STs) in the subnanosecond range remained poorly investigated.

The difficulties of such investigations were primarily determined by the complexity of creating the equipment for exciting and diagnosing STs. Meanwhile, when a semiconductor is excited with highintensity picosecond electric-field or electron-beam pulses, a number of effects may arise, which may lead to an increase in the intensity and efficiency of lasing. Blooming of the passive semiconductor region and a decrease in both the heating of the active region and radiation losses during the lifetime of nonequilibrium charge carriers can be considered as such effects.

Significant successes in the development of highpower subnanosecond sources of electromagnetic fields and dense electron flows [3-5] determined the new possibilities in the formation of picosecond pulses with required parameters and, consequently, creation of new trends in studies of the physical processes in highly excited semiconductors [6, 7]. New test benches equipped with broadband instrumentation were developed to excite and diagnose the emission of STs [7-10]. The following most important results were obtained at these benches: (i) a record peak radiation intensity of $3 \times 10^{7} \mathrm{~W} / \mathrm{cm}^{2}$ and a peak power of $10 \mathrm{MW}$ were achieved for electron-beam-excited semiconductor lasers in the picosecond range on a CdS target in the green spectral region $(\lambda=522 \mathrm{~nm})$;

(ii) as the pressure in the diode chamber increases from $10^{-1}$ Torr to atmospheric pressure, a consecutive decrease in the durations of the electron-beam current and laser radiation of the target from hundreds to tens of picoseconds was observed and the light-pulse shape reproduced the current-pulse shape; superluminescence $(\lambda=480 \mathrm{~nm})$ that was excited by runaway electrons was achieved at atmospheric pressure on a $\mathrm{ZnSe}$ target;

(iii) when a $\mathrm{ZnCdS}$ solid-solution target was excited by a pulsed electric field, laser oscillation was sequentially observed at three spectral lines of 509, 480, and $469 \mathrm{~nm}$ during motion of a streamer discharge from one surface of the semiconductor target to the opposite surface.

A further decrease in the duration of picosecond laser pulses and an increase in their power are related to the necessity of increasing the source power density. As an example, the effective conversion of the electron-beam (EB) energy into laser radiation in the picosecond range requires a power density that may exceed $10^{9} \mathrm{~W} / \mathrm{cm}^{2}$.

One of the methods for solving this problem is high-frequency modulation of the electric field. Exciting an ST using an EB, whose energy increase and 


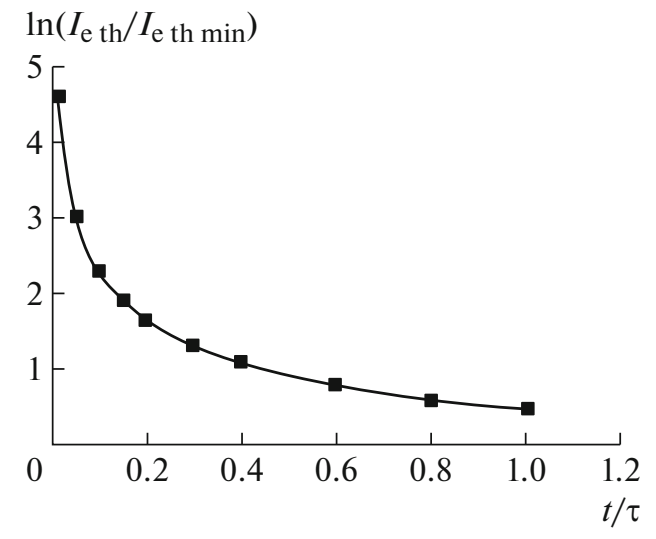

Fig. 1. The change in the EB threshold density as a function of the ST excitation time.

modulation occur due to a microwave field in one or several cavity resonators, was proposed in 1968 and performed in 1971 [11]. Using a four-resonator electron accelerator with preliminary grouping of the electron flow, generation of a microwave pulse of IR laser radiation was obtained on a GaAs ST, which was cooled with liquid nitrogen. According to rough estimates, the lasing power in this short peak was $\sim 10 \mathrm{~kW}$. Unfortunately, technical and experimental difficulties limited the further development of these experiments.

This paper describes the equipment and techniques that determine the possibility of reducing the EB threshold power and the formation of high-intensity laser pulses in the picosecond range via high-frequency modulation of high-voltage pulses by a nonlinear gyromagnetic ferrite line with adjustable saturation. The principle of reducing the EB threshold power is based on the existence of a threshold energy $E_{\mathrm{th}}$, at which lasing is initiated. The value of $E_{\mathrm{th}}$ at a specified parameters of the ST depends on three main parameters: the accelerating voltage $U$, the current density $i_{\mathrm{e}}$, and the EB duration $t_{\mathrm{e}}$. As the duration $t_{\mathrm{e}}$ increases and the accelerating voltage $U$ remains constant, the current density $i_{\mathrm{e}}$ at which lasing arises correspondingly decreases. It is obvious that if the SM is excited by a modulated EB or a train of ultrashort pulses with a duration $t_{\mathrm{p}}<t_{\mathrm{e}}$ and the excitation energy $E_{k} \leq E_{\mathrm{th}}$, in this case, the threshold energy can be achieved upon fulfillment of the conditions $E_{\mathrm{th}}=\sum_{k=0}^{n} E_{k}$ and $\tau \geq t_{\mathrm{e}} \geq T$, where $T \approx 2 t_{\mathrm{p}}$ is the period of the accelerating-voltage modulation frequency and $\tau$ is the lifetime of nonequilibrium charge carriers.

After the threshold energy is reached, a lasing pulse is initiated with the shape that reproduces the shape of the modulated EB pulse. If it is necessary to reduce the laser pulse duration by several times with maintenance of its shape, intensity, and efficiency of the EB energy conversion into laser radiation, it is necessary to excite the target with EB pulses, the number of which is increased, while the duration is shorter by the same number of times. If the above conditions are met, the radiation delay, duration, and intensity can be varied by changing the parameters of the exciting pulse.

Let us consider these conditions in more detail. Lasing occurs when the amplification compensates for all losses in the optical resonator of the ST. The gain $g$ in turn depends on the concentration $n$ of nonequilibrium charge carriers (NCC). In the pulsed operating mode, $n$ can be estimated from the volume rate $G$ of $\mathrm{NCC}$ generation and the duration $t_{\mathrm{e}}$ of the EB action on the ST [1]:

$$
\begin{gathered}
n \approx G \tau\left(1-e^{-t_{e} / \tau}\right), \\
G=i_{\mathrm{e}} U / 3 E_{\mathrm{g}} z_{\mathrm{a}} q,
\end{gathered}
$$

where $i_{\mathrm{e}}$ is the EB current density with account for losses during its interaction with the target surface; $U$ is the accelerating voltage; $3 E_{\mathrm{g}}$ is the energy expended on ionizing one electron-hole pair; $E_{\mathrm{g}}$ is the semiconductor energy-gap width; $z_{\mathrm{a}}$ is the active EB penetration depth; and $q$ is the electron charge. It follows from (1) and (2) that at the threshold concentration $n_{\text {th }}$, the threshold power density (intensity) of the EB is defined by the formula

$$
I_{\mathrm{e} \text { th }}=i_{\mathrm{e} \text { th }} U \approx I_{\mathrm{eth} \min }\left(1-e^{-t_{\mathrm{e}} / \tau}\right)^{-1},
$$

where $I_{\mathrm{e}}$ th min is the minimum value of the threshold power density, which is reached at $t_{\mathrm{e}} \approx \tau$. As $I_{\mathrm{e}}$ increases, lasing shifts to the onset of the exciting EB pulse, reproducing its shape in the first approximation. A decrease in the lasing threshold with increasing $t_{\mathrm{e}}$ can be estimated from the formula

$$
N \approx\left(1-e^{-t_{\mathrm{c} 2} / \tau}\right) /\left(1-e^{-t_{\mathrm{c} 1} / \tau}\right) .
$$

As an example, for $t_{\mathrm{e} 1} / \tau=0.1$ and $t_{\mathrm{e} 2} / \tau=1$, the lasing threshold for $t_{\mathrm{e} 2}$ in comparison to $t_{\mathrm{e} 1}$ must be lower by a factor of $\sim 7$. The lifetime in cadmium sulfide is $\tau \sim 1 \mathrm{~ns}$, from which the equivalent modulation frequency follows: $F \approx 0.5 / t_{\mathrm{e} 1}=5 \mathrm{GHz}$. The change in the threshold power density as a function of the excitingpulse duration is shown in Fig. 1.

The schematic diagram of the complex for investigating an ST in the mode of excitation by the EB with the use of high-frequency modulation of the accelerating voltage is shown in Fig. 2. A high-voltage pulse from the RADAN-303 generator enters the first stage of a two-stage amplitude-to-time converter 1, which was described in detail in [12]. At the first stage, the inductive-capacitive method for the voltage-pulse compression with simultaneous shortening of the accelerating-voltage pulse front to $200 \mathrm{ps}$ is used. After 


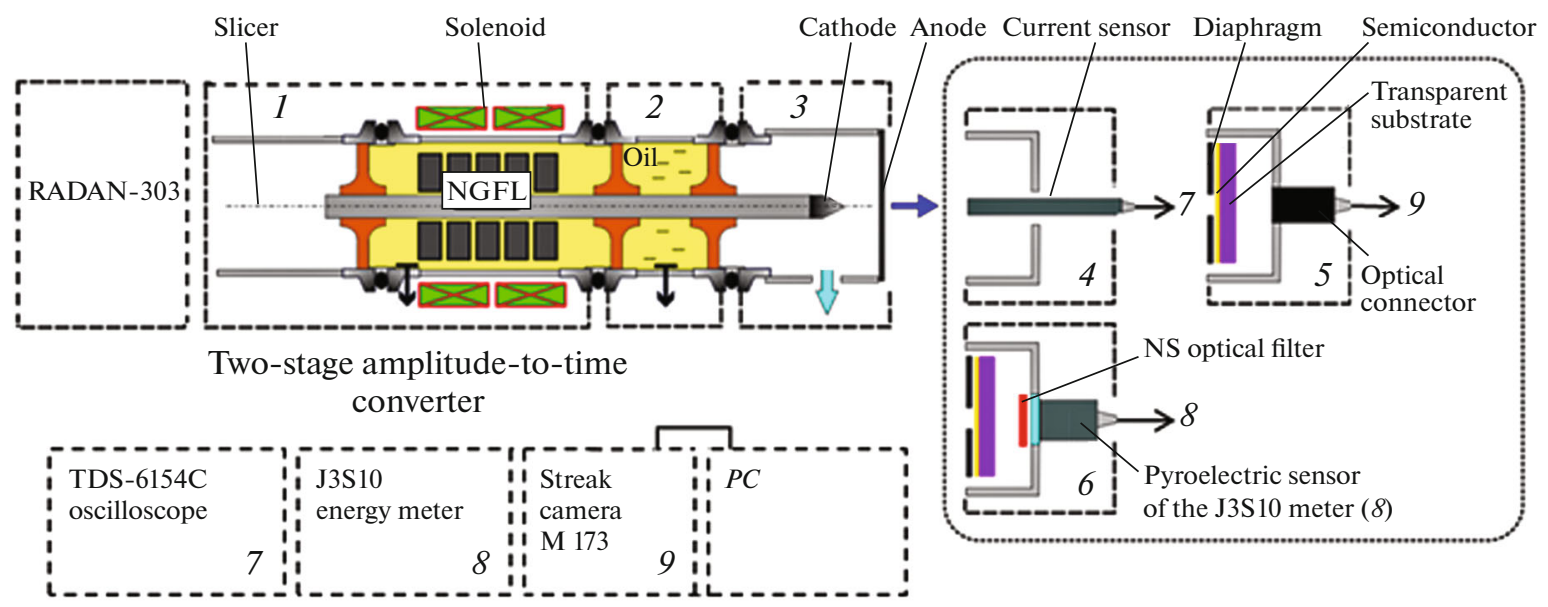

Fig. 2. The diagram of the complex for excitation and diagnostics of radiation from a semiconductor target excited with a modulated electron beam: (RADAN-303) high-voltage pulse generator; (1) hybrid two-stage converter of a high-voltage pulse from the generator; $(N G F L)$ nonlinear gyromagnetic ferrite line; (2) transmission line with a voltage divider; (3) diode chamber; (4-6) attachments with semiconductor targets and sensors for measuring the EB current and characteristics of laser radiation; and (PC) personal computer.

the first compression stage, the pulse voltage increases from -150 to $-220 \mathrm{kV}$.

The second stage is a nonlinear gyromagnetic ferrite line (NGFL) with adjustable saturation. The parameters of such a line and a certain value of the magnetization field provide the high-frequency modulation of the pulse top with a frequency of up to $5 \mathrm{GHz}$ and a modulation depth of at least $50 \%$. Structurally, the ferrite line is a coaxial line (with outer/inner diameters of $18 / 8 \mathrm{~mm}$ ) with oil insulation at a pressure of 5 atm, into which NiZn ferrite rings are built. The ferrite assembly is made of VNP rings with outer/inner diameters of $16 / 10 \mathrm{~mm}$. The total length of the assembly is $\sim 400 \mathrm{~mm}$.

The line operated in the biasing mode using an external solenoid that was mounted over the ferrite line. The magnetic field was set by a DC value of a solenoid-supplying programmable source, thus providing an opportunity to optimize the parameters of the output pulse within a certain range. This technical solution allows one to additionally reduce the voltagepulse rise time.

For an output voltage amplitude of $-200 \mathrm{kV}$ and a biasing current of $\sim 55 \mathrm{kA} / \mathrm{m}$, a pulse rise time of $\sim 85 \mathrm{ps}$ was obtained. The absence of a prepulse before the main pulse at the output of the ferrite line is another important point, which is always formed in gas switches, as, e.g., in the first stage due to the presence of a capacitance between the electrodes of the spark gap; as a result, this may unpredictably affect the parameters of the charged-particle beam.

The pulse amplitude and shape are controlled with a broadband capacitive sensor, which is built into transmission line 2. Diode 3 with a graphite cathode operating in the explosive-emission mode is the source of electrons. A diaphragm (anode) with an opening closed with an $\mathrm{Al}-\mathrm{Be}$ foil is installed at a distance of 3-6 $\mathrm{mm}$ behind the cathode. Different attachments (4-6) were used to study the characteristics of the diode and semiconductor targets in the picosecond range. The required attachment was installed at a distance of a few millimeters from the diode flange before the beginning of the experiment. The current density was measured with a broadband sensor 4 [13].

The spectrum, radiation dynamics, and light pulse shape were registered using an attachment 5 with a connector and a microlens, which focused the radiation from the ST to the input of a fiber-optic cable (FOC). The laser energy was controlled with a J3S10 pyroelectric detector $(8)$. The FOC output was connected to a streak camera 9 (model 173, Cordin) or a spectrometer (model MS-257, Oriel Instruments). Signals from the output of the streak camera were fed to the personal computer (PC) input and observed on its monitor. The delay time of a light pulse relative to

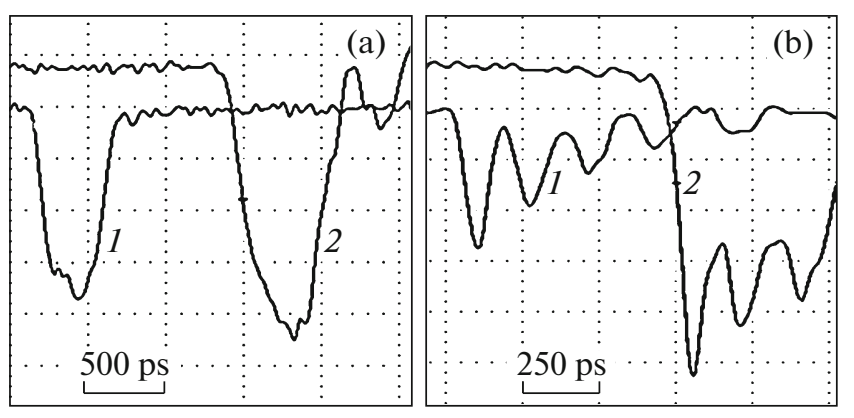

Fig. 3. Oscillograms of EB current (1) and voltage (2) pulses: (a) without and (b) with modulation of the pulse top. 

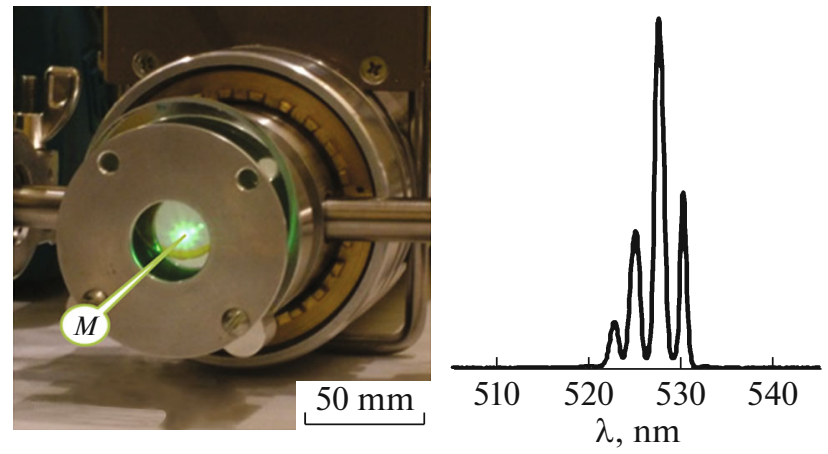

Fig. 4. (a) The diode chamber with the laser target $T$ (cadmium sulfide) and (b) the lasing spectrum of the target.

the synchronization pulse was adjusted by the FOC length. The shape of the light pulse was determined from the streak-photograph profile. The signal was attenuated by NS optical filters that were installed in front of the detectors. A Tektronix TDS-6154C oscilloscope (with a bandwidth of $15 \mathrm{GHz}$ ) was used to register voltage and current pulses. Figure 3 shows current (curves 1) and voltage (curves 2) waveforms without (Fig. 3a) and with modulation (Fig. 3b). It is seen that after the high-voltage pulse was modulated, the rise times of the current and voltage pulses decreased by a factor of more than 2 .

The semiconductor compounds, which are used to manufacture lasers with excitation by EBs, include binary compounds: $\mathrm{ZnS}, \mathrm{ZnO}, \mathrm{ZnSe}, \mathrm{CdS}, \mathrm{CdSe}$, and their solid solutions: $\mathrm{ZnCdS}, \mathrm{ZnCdSe}$, and CdSSe, which radiate from the UV to the IR spectral region. Of these compounds, the physical characteristics of cadmium sulfide have been studied in the most detail. Therefore, at the first stage of our study, the used STs were made of a single-crystal CdS film that was fixed on a sapphire substrate. The film thickness was selected taking the active penetration depth of the EB into account. Reflecting coatings that formed an optical resonator were deposited on the film planes: a metal coating from the side of the EB and a multilayer dielectric coating on the opposite side. The semiconductor target was manufactured according to the technology corresponding to the conditions for high-efficiency lasing [14, 15]. The diameter of the electron spot on the ST was $0.75 \mathrm{~mm}$. The diode chamber with the laser target of cadmium sulfide and a typical emission spectrum in the lasing mode are shown in Fig. 4.

The experimental technique consisted in recoding the oscillograms of accelerating-voltage pulses, the EB current, and streak photographs of laser radiation with their subsequent processing and analysis of the results. Figure 5 shows the changes in the power density of EB pulses (curves 1) calculated from the current and voltage waveforms without modulation and with high-frequency modulation, as well as the profiles (curves 2) and streak photographs of laser pulses.

Let us consider the results. In Fig. 5a, laser radiation (2) arises with a delay at the front of an EB pulse (1) and then reproduces its shape quite accurately. Some tailing at the trailing edge of the light pulse can be explained by the decay of spontaneous emission. Figures $5 \mathrm{~b}$ and $5 \mathrm{c}$ show an increase in the delay of laser radiation, and the laser-pulse shape (2) does not always corresponds to the shape of the EB pulse (1). The maximum intensity of laser radiation $J_{\mathrm{Lm}}$ in the cases of Figs. 5a-5c was, respectively, $J_{\mathrm{Lm}(\mathrm{a})} \approx 4 \times 10^{7} \mathrm{~W} / \mathrm{cm}^{2}$, $J_{\mathrm{Lm}(\mathrm{b})} \approx 1.9 \times 10^{7} \mathrm{~W} / \mathrm{cm}^{2}$, and $J_{\mathrm{Lm}(\mathrm{c})} \approx 10^{7} \mathrm{~W} / \mathrm{cm}^{2}$. In all of these three cases, the calculated NCC concentration at the points of onset of lasing was $n_{\mathrm{th}} \approx 5 \times$ $10^{18} \mathrm{~cm}^{-3}$.

The optical gain corresponding to this concentration must be equal to all losses reduced to the length of the optical resonator. In our case, at high accelerating
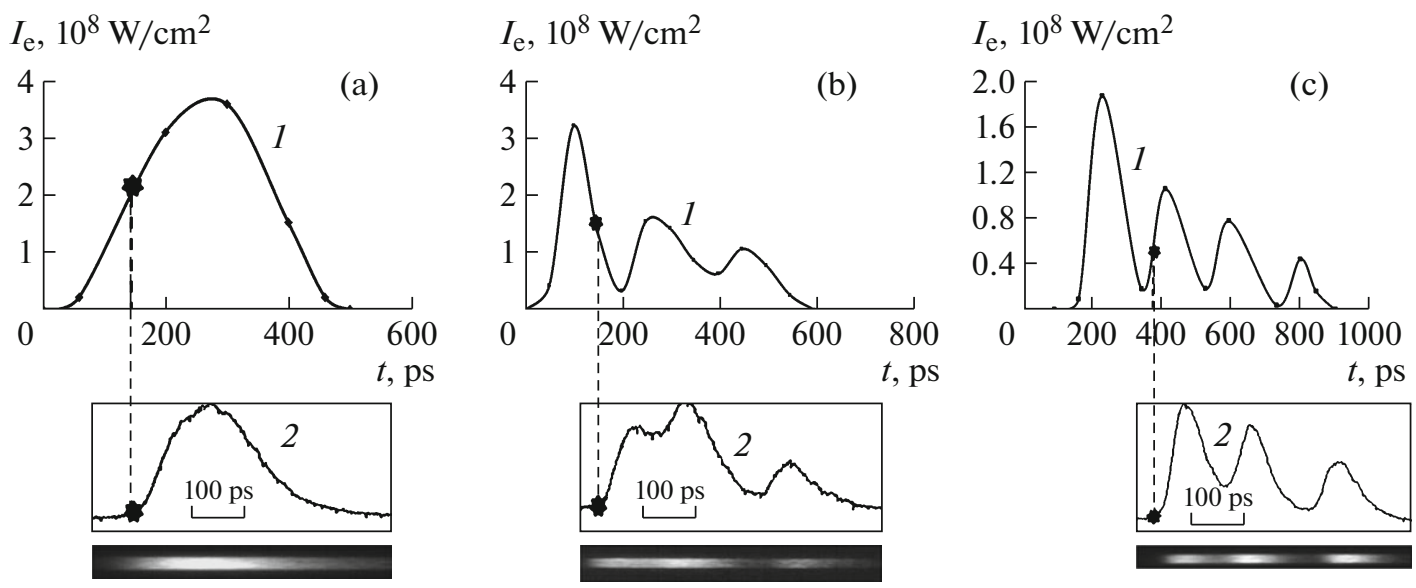

Fig. 5. (1) The changes in the EB power density within the duration of voltage pulses without (a) and with high-frequency modulation (b, c), the profiles (2) and streak photographs of laser pulses. An asterisk marks the onset of lasing. 


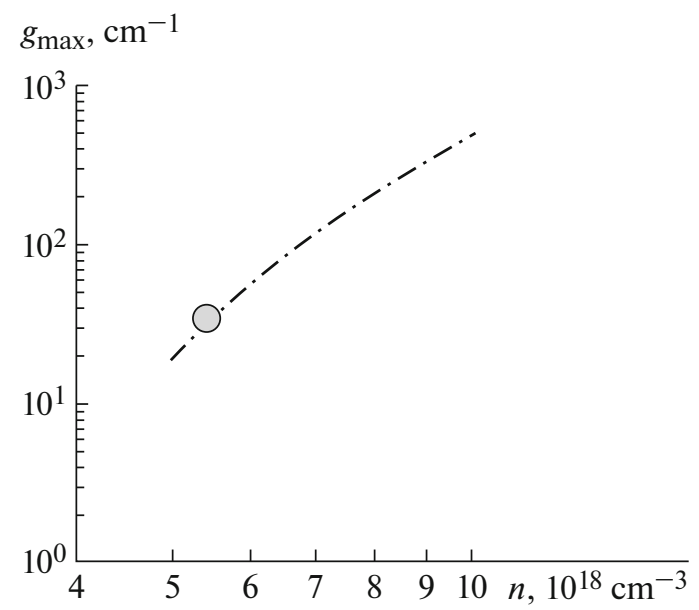

Fig. 6. The dependence of the maximum optical gain $g_{\max }$ on the NCC concentration for CdS at $300 \mathrm{~K}$ (calculated in [17]). The marked point on the curve corresponds to the results obtained in this study.

energies and EB current densities, the total losses in the optical resonator were mainly determined by the losses on the mirrors and were $\sim 25-30 \mathrm{~cm}^{-1}$ [7]. These data coincide well (see Fig. 6) with the results of theoretical calculations of the dependence of the optical gain factor $g_{\max }$ on the NCC concentration taking the electron-hole correlation for CdS at $T=300 \mathrm{~K}$ into account [16]. It is seen from the graph in Fig. 6 that the coefficient $g_{\max } \approx 30 \mathrm{~cm}^{-1}$ corresponds to the concentration $n \approx 5 \times 10^{18} \mathrm{~cm}^{-3}$. The calculation technique with consideration for the EB pulse shape was presented in more detail in [17].

Thus, it follows from these results that the excitation of an ST with an EB modulated by a high frequency, provided that the duration of the exciting pulse $t_{\mathrm{e}}$ is less than the NCC lifetime $\tau$ and exceeds the period of high-frequency oscillations $T$, provides a sufficiently wide range of the possibilities to control laser radiation of a semiconductor target, namely:

- it allows a decrease in the EB threshold power density at which lasing is initiated (Fig. 5);

- allows changing the duration of laser pulses by changing the modulation frequency;

- it is possible to adjust the shape of a laser pulse (Fig. 5b) and the time delay of a laser pulse relative to the exciting EB pulse (Fig. 5).

It should be noted that the high-frequency modulation of the EB using the methods of nonlinear electrodynamics is reasonable primarily when the maximum power density is reached and a further reduction of the pulse duration with the preservation of the efficiency of the EB energy conversion into laser radiation is possible only via accumulation of the prethreshold NCC density within a time that does not exceed the lifetime.
These results confirmed the possibility of using the developed complex of equipment for further studies in the field of increasing the intensity and reducing the duration of laser pulses in the picosecond range. The task of the subsequent stages of this study is to further increase the EB frequency and power using the methods of nonlinear electrodynamics and research the application of this method to obtaining high-intensity picosecond pulses on laser targets of other semiconductors.

\section{FUNDING}

This study was performed within the framework of the Program no. 10 of the Presidium of the Russian Academy of Sciences.

\section{OPEN ACCESS}

This article is distributed under the terms of the Creative Commons Attribution 4. International license (http://creativecommons.org/licenses/by/4.0/), which permits unrestricted use, distribution, and reproduction in any medium, provided you give appropriate credit to the original author(s) and the source, provide a link to the Creative Commons license, and indicate if changes were made.

\section{REFERENCES}

1. Bogdankevich, O.V., Darznek, S.A., and Eliseev, P.G., Poluprovodnikovye lazery (Semiconductor Lasers), Moscow: Nauka, 1976.

2. Kryukova, I.V., Fizicheskie protsessy $v$ poluprovodnikovykh impul'snykh lazerakh s nakachkoi elektronnymi puchkami (Physical Processes in Semiconductor Pulse Lasers with Electron-Beam Pumping), Moscow: Bauman Moscow State Technical Univ., 2009.

3. Mesyats, G.A., Impul'snaya energetika i elektronika (Pulse Power and Electronic Engineering), Moscow: Nauka, 2004.

4. Mesyats, G.A. and Yalandin, M.I., Usp. Fiz. Nauk, 2005, vol. 175, no. 3, p. 225.

5. Nasibov, A.S., Berezhnoi, K.V., Shapkin, P.V., Reutova, A.G., Shunailov, S.A., and Yalandin, M.I., Instrum. Exp. Tech., 2009, no. 1, p. 65. https://doi.org/10.1134/S0020441209010102

6. Mesyats, G.A., Nasibov, A.S., Shpak, V.G., Shunailov, S.A., and Yalandin, M.I., J. Exp. Theor. Phys. (JETP), 2008, vol. 106, no. 6, p. 1013.

7. Berezhnoi, K.V., Bochkarev, M.B., Danielyan, G.L., Nasibov, A.S., Reutova, A.G, Shunailov, S.A., and Yalandin, M.I., Kvantovaya Elektron., 2012, vol. 42, no. 1, p. 34 .

https://doi.org/10.1070/QE2012v042n01ABEH000000

8. Yalandin, M.I., Bochkarev, M.B., Shunailov, S.A., Sadykova, A.G., Nasibov, A.S., Bagramov, V.G., Berezhnoi, K.V., and Vasil'ev, B.I., Instrum. Exp. Tech., 2017, vol. 60 , no. 5 , p. 710 .

https://doi.org/10.1134/S0020441217050116 
9. Nasibov, A.S., Danielyan, G.L., Bagramov, V.G., Berezhnoi, K.V., and Shapkin, P.V., Bull. Lebedev Phys. Inst., 2011, vol. 38, no. 4, p. 101.

10. Bagramov, V.G., Danielyan, G.L., Nasibov, A.S., Podvyaznikov, V.A., Tasmagulov, I.D., and Chevokin, V.K., Bull. Lebedev Phys. Inst., 2018, vol. 45, no. 4, p. 108.

11. Bogdankevich, O.V., Korolev, S.V., Nasedkin, A.A., Olikhov, I.M., and Petrov, D.M., Kvantovaya elektronika (Quantum Electronics), Basov, N.G., Ed., Moscow: Sovetskoe Radio, 1971, no. 4, p. 97.

12. Ulmaskulov, M.R., Mesyats, G.A., Sadykova, A.G., Sharypov, K., Shpak, V.G., Shunailov, S., and Yalandin, M.I., Rev. Sci. Instrum., 2017, vol. 88, no. 4, p. 045106.

13. Reutova, A.G., Sharypov, K.A., Shpak, V.G., Shunailov, S.A., and Yalandin, M.I., Proc. 15th Int. Symposium on High Current Electronics, Tomsk: Institute of
High Current Electronics Siberian Branch Russ. Acad. Sci., 2008, p. 111.

14. Kozlovskii, V.I., Nasibov, A.S., Pechenov, A.N., Popov, Yu.M., Talenskii, O.N., and Shapkin, P.V., Kvantovaya Elektron., 1977, vol. 4, no. 2, p. 351.

15. Daneu, V., DeGloria, D.P., Sanchez, A., and Osgood, R.M., Appl. Phys. Lett., 1986, vol. 49, no. 10, p. 8.

16. Nabiev, R.F. and Popov, Yu.M., Laser-Cathode Ray Tubes, New York: Nova Science Publ., 1996, vol. 221, p. 1.

17. Nasibov, A.S., Bagramov, V.I., Berezhnoi, K.V., Bochkarev, M.V., Sadykova, A.G., Tasmagulov, I.D., and Sharypov, K.A., Bull. Lebedev Phys. Inst., 2019, vol. 46, no. 1 , p. 1 .

Translated by A. Seferov 